\title{
DISCUSSÕES BIOÉTICAS EM PESQUISA ENVOLVENDO SERES HUMANOS: PROBLEMATIZAÇÕES A PARTIR DAS PERSPECTIVAS NORMATIVAS
}

\author{
Bruna Bottero Corrêa* \\ Maria Claudia Crespo Brauner ${ }^{* *}$
}

\begin{abstract}
RESUMO
A tendência normativa direcionada à bioética pode vir a retirar direitos consolidados pelas resoluções até então vigentes no Brasil. Diante disso, os Comitês de Ética em Pesquisa (CEPs) podem exercer papel fundamental na preservação da bioética. Isto porque são órgãos que permitem a prática democrática preservando a dinâmica necessária aos requisitos em pesquisa, em detrimento da comumente rigidez legislativa. No entanto, mesmo nesse contexto, alguns fatores importantes precisam ser observados, como a questão do conflito de interesses e a responsabilização institucional. Algumas tendências em propostas legislativas pretendem flexibilizar essas exigências colocando em risco as garantias asseguradas pela longa trajetória de entendimento da bioética no país. Além disso, tende a comprometer o próprio fluxo democrático deslocando-o para uma aparente ampliação da autonomia. O objetivo do presente artigo é realizar um breve estudo comparado entre as resoluções de 196/96 e 466/12 do Conselho Nacional de Saúde Brasileiro e da síntese comparativa analisar o projeto de lei $\mathrm{n}^{\circ} 200$ proposto pelo Senado Federal que trata da pesquisa clínica, a fim de identificar as alterações mais problemáticas envolvendo participantes e o sistema nacional de controle das pesquisas. A metodologia adotada é a análise bibliográfica. Justifica-se a importância da perspectiva adotada para a abordagem do tema bioética por se entender relevante para o mundo jurídico compreender a relação existente entre a
\end{abstract}

\footnotetext{
"Mestranda em Direito e Justiça Social no Programa de Pós-Graduação em Direito da Universidade Federal do Rio Grande (FURG).

** Doutora em Direito pela Université de Rennes I - França (1992); Pós-Doutora na Universidade de Montreal 1 - Canadá. Coordenadora do Mestrado em Direito e Justiça Social da Universidade Federal do Rio Grande (FURG).
} 
problemática regulamentação da pesquisa e os interesses da sociedade. Igualmente, não foram encontrados trabalhos problematizando os direitos garantidos aos participantes e a importância dos CEPs, através do método de análise comparativa.

PALAVRAS-CHAVE: pesquisa envolvendo seres humanos; análise comparativa das resoluções 196/1996 e 466/2012; Comitês de Ética em Pesquisa; proposta legislativa 200/2015; tendências.

\begin{abstract}
The normative tendency directed to bioethics may lead to the withdrawal of rights consolidated by the resolutions that were in force in Brazil. Faced with this, the Research Ethics Committees (CEPs) can play a fundamental role in the preservation of bioethics. This is because they are organs that allow the democratic practice preserving the dynamics necessary to the requirements in research, to the detriment of the commonly rigid legislation. However, even in this context, some important factors need to be observed, such as the issue of conflict of interest and institutional accountability. Some tendencies in legislative proposals intend to make these requirements more flexible, jeopardizing the guarantees guaranteed by the country's long-standing understanding of bioethics. Moreover, it tends to compromise the democratic flow itself, shifting it towards an apparent enlargement of autonomy. The purpose of this article is to conduct a brief comparative study between the 196/96 and 466/12 resolutions of the Brazilian National Health Council and the comparative synthesis to analyze the Bill 200 proposed by the Federal Senate that deals with clinical research, To identify the most problematic changes involving participants and the national system of research control. The methodology adopted is the bibliographic analysis. The importance of the perspective adopted for the bioethical approach is justified because it is considered relevant for the legal world to understand the relationship between the problematic regulation of research and the interests of society. Likewise, no papers were found problematizing the rights guaranteed to the participants and the importance of the CEPs, through the method of comparative analysis.
\end{abstract}

KEYWORDS: Research involving human beings; Comparative analysis of resolutions 196/1996 and 466/2012; Ethics Committees in Research; Legislative proposal 200/2015; tendencies. 


\section{INTRODUÇÃO}

A ética em pesquisa envolvendo seres humanos é tema em constante debate e motivo de tensas disputas, especialmente no que tange aos interesses na área de pesquisa clínica. O presente ensaio procura abordar alguns aspectos normativos que regulamentam essas pesquisas, relacionando-os com os estudos na área científica da bioética e das teorias éticas principiológicas.

O trabalho está dividido em duas partes. Em um primeiro movimento, procura-se destacar alguns recortes históricos do desenvolvimento da ética em pesquisa no Brasil. Busca-se em seguida, iniciar um breve estudo comparativo das resoluções 196/96 e 466/96 do Conselho Nacional de Saúde, salientando-se a importância dos comitês de ética em pesquisa no seu papel de controle, proteção e pedagogia ético-bioética. Por fim, no terceiro e último movimento, o objetivo é demonstrar as tendências em alterações ético-normativas a partir do projeto de lei proposto pelo Senado Federal número 200 de 2015.

A técnica de pesquisa adotada é a análise bibliográfica e documental. Utilizou-se para tanto de artigos científicos nas áreas biomédica e ética, documentos legislativos, pareceres de organizações e da Comissão Nacional de Ética em Pesquisa (CONEP).

Os comitês regionais de ética em pesquisa (CEPs) são colegiados independentes, consultivos, deliberativos e educativos. Sua composição deve respeitar a multidisciplinaridade e prezar pela transdiciplinaridade, através da composição e atuação democrática de seus membros. À Comissão Nacional de Ética em Pesquisa (CONEP) são atribuídas as mesmas competências acrescentando-se a instância normativa e a vinculação direta ao Ministério da Saúde.

A partir de um estudo comparativo normativo, busca-se destacar os focos críticos que possivelmente mais afetam os direitos adquiridos com relação à ética em pesquisa envolvendo seres humanos, destacando dentre eles, a proteção do participante e a importância dos CEPs. Além disso, pretende-se investigar os posicionamentos de algumas publicações na área do tema, a partir da análise bibliográfica reunida, visando destacar criticamente o que parecem ser alguns caminhos que estão sendo tomados pela bioética contemporânea. 


\section{A PESQUISA ENVOLVENDO SERES HUMANOS NO BRASIL}

A pesquisa com seres humanos no Brasil possui uma considerável trajetória e experiência no que se refere a sua regulamentação e enquadramento nos parâmetros da bioética, a partir de referências mundiais e estruturas democráticas. De acordo com Goldim (2002), no Brasil, a intenção de controlar a pesquisa desenvolvida envolvendo seres humanos se inicia em 1975 quando o país adere à primeira versão da Declaração de Helsinki (1964), resultante do esforço internacional em estabelecer diretrizes de controle e reflexão geral sobre os princípios éticos e humanistas fundamentais. Estes passaram a ser considerados nas pesquisas, em especial na área da pesquisa clínica, a partir das discussões do pósguerra.

A Constituição Federal de 1988 preceitua as disposições que orientam o Ministério da Saúde e demais sistemas a ele submetidos, que por sua vez integram um organismo especializado em discutir as questões éticas na pesquisa, o reconhecido e renomado sistema $\mathrm{CEP} / \mathrm{CONEP} / \mathrm{CNS} / \mathrm{MS}$. Esse sistema é tido como referência mundial, com destaque dentre os países sul-americanos.

Os conselhos regionais ou locais (CEPs), com seu comitê nacional (CONEP) ligado ao Conselho Nacional (CNS) e ao próprio Ministério da Saúde (MS), juntos, formam a articulação responsável pela regularização da ética em pesquisa no país. O sistema divulga os entendimentos atinentes e publica resoluções, em especial no que concerne a proteção dos participantes, sendo responsável ainda pelo registro, divulgação e controle dos projetos em andamento.

Dentre as resoluções, destacam-se a 196/1996 e a 466/2012 do Conselho Nacional de Saúde, a primeira revogada pelo advento da segunda, tendo em vista o aprimoramento dos seus critérios. Essas alterações foram realizadas com base no trabalho que vem sendo construído pelos conselhos avaliadores desde 1988 e aprofundado pelo desenvolvimento do referido sistema, formalmente apresentado na resolução de 2012. A partir de um trabalho inicial, voltado para a ética em pesquisa clínica e para a assistência médica, foram desenvolvidos parâmetros éticos e legais que passaram a contemplar as diferentes áreas científicas. 
Embora a alcunha bioética tenha sido criada com o intuito de englobar o relacionamento do homem com e fazendo parte de uma biologia ampla, a partir da análise da resolução 196/96 é possível perceber a influência da bioética dos princípios: da beneficência, da autonomia e da não maleficência, e da justiça, estes que por sua vez permeiam os demais requisitos, legais e políticos, a serem destacados no presente estudo. Segundo esse documento, para situar o tema desse escrito, pesquisa envolvendo seres humanos é a pesquisa que, individual ou coletivamente, tenha como participante o ser humano, em sua totalidade ou partes dele, e o envolva de forma direta ou indireta, incluindo o manejo de seus dados, informações ou materiais biológicos.

Para Barbosa, Corrales e Silbermann (2014) a pesquisa envolvendo seres humanos pode ser dividida conceitualmente na pesquisa com e em seres humanos. A pesquisa com seres humanos envolve a interação comunicativa entre $o$ pesquisador $e$ participante, em sua maior parte aplicada nas áreas de ciências sociais e humanas. Já a pesquisa em seres humanos está mais diretamente relacionada à biomedicina, no manejo de material genético e do próprio corpo humano na investigação de tratamentos médicos e instrumentais, adentrando mais o ser.

Da análise regulamentadora resulta que, a pesquisa envolvendo seres humanos tem caráter excepcional. Nesse sentido, pode ser considerado um princípio primordial, expresso no item III. 2, alínea $c$, da resolução 466/12 que indica que ela deve ser realizada "somente quando o conhecimento que se pretende obter não possa ser obtido por outro meio", não devendo, em regra, ser adotada. Dentre os demais requisitos, a resolução prevê principalmente os direitos e deveres que dizem respeito aos participantes, pesquisadores e instituições, públicas ou privadas.

O controle bioético, no seu plano ideal, estaria autorizado e obrigado a avançar na averiguação sobre toda a pesquisa científica, seja ela aplicada a partir das ciências sociais, sociais aplicadas, bem como nas humanas propriamente ditas e inclusive nas exatas. É possível insinuar que a área médica reproduz a preocupação ética há mais tempo na pesquisa brasileira, por tratar mais diretamente de pesquisas com maior cunho invasivo envolvendo seres humanos. Apesar dos especiais avanços protetivos e regulatórios sobre as 
pesquisas desse tipo no contexto brasileiro, o estudo de Santana e Faria (2013) reconhece que ainda não há uma exigência rigorosa em algumas áreas da ciência visando proteger os sujeitos, e a preocupação por parte dos conselhos editorais, que publicam as pesquisas, ainda é principiante.

Porém, em uma análise crítica especulativa é possível sugerir que o fato de existirem trabalhos acadêmicos dedicados a verificar se as pesquisas estão respeitando os princípios éticos a partir da preocupação de todos os envolvidos, nos diferentes ramos da ciência, demonstra um grau positivo no quadro atual. Essa indicação pode ser um adiantamento na problematização do tema, ainda que através de um trabalho gradual em termos de proteção ética. Isto se deve ao fato de que garantias vêm sendo asseguradas expressamente e se ampliam com a renovação das normativas, estudos e órgãos atinentes. Aliamse a estas previsões, o comprovado estatisticamente, de acordo com o estudo de Zoboli (2004), aumento do número de conselhos de ética em pesquisa nas diversas áreas, nas universidades e em diversas instituições do país.

\subsection{Breves considerações a partir de uma análise comparativa das Resoluções 196/1996 e 466/2012 do Conselho Nacional de Saúde}

Em termos éticos e jurídicos, um estudo comparado entre as duas resoluções, a anterior e a vigente, revela que equilibradamente a resolução 466/12 realiza um movimento de ampliação da autonomia, ao mesmo tempo em que aumenta os direitos de proteção dos participantes. $\mathrm{Na}$ análise comparativa entre os aspectos éticos da pesquisa envolvendo seres humanos e seus requisitos, na resolução 466/12, ao participante é permitida uma maior autonomia ou liberdade de escolha, sem afetar o equilíbrio entre os membros da pesquisa. Isto se deve evidentemente, à questão da vulnerabilidade daqueles, que de uma maneira mesmo que consentida, se submetem ao estudo.

Ao participante é assegurado, a depender da sua vontade, após iniciada a pesquisa, permanecer nela ou não, desde que haja manifestação livre e esclarecida, de maneira expressa. A indicação do consentimento expresso foi acrescida pela nova resolução, complementando a disposição anterior que exigia o consentimento 
livre e esclarecido sem mencionar ou exigir a sua documentação. Observa-se que essa modificação, dentre outras, podem ser oriundas das experiências práticas dos envolvidos com as avaliações éticas e da questão da exigibilidade dos direitos e deveres consentidos quando da colaboração para a produção científica, na esfera judicial.

A manifestação da vontade do participante se traduz no documento, elaborado pelo pesquisador, denominado Termo de Consentimento Livre e Esclarecido (TCLE). Juridicamente, é o documento inequívoco das condições acordadas entre pesquisador e participante. Para Goldim (2002), o TCLE tem sido um dos temas mais discutidos na bioética contemporânea. Para o estudioso, a vinculação do consentimento com a autonomia tem dado um viés muito individualista à questão. Para ele, a confiança recíproca e o compartilhamento de informações é o fundamental para garantir a ética em pesquisa, sugerindo uma socionomia, mediante um envolvimento e cuidado estabelecidos nessa relação.

Já para o clássico posicionamento de Engelhardt Jr. (1998), a questão ou princípio do consentimento ou da autonomia, pode tomar outros contornos. Embora o posicionamento de $\mathrm{H}$. Tristam Engelhardt Jr. seja considerado como radical, representando o paradigma libertário norte-americano centrado na valoração da autonomia, sua demonstração pode ser uma saída para a bioética contemporânea. Ele conduz ao entendimento de que o contexto de uma norma geral, injustificável a partir da racionalidade, não se sustenta diante da complexidade da sociedade pluralista.

A importância crucial da substituição dessa ideia por uma que defenda a negociação e o acordo mútuo, mostra-se de fato adequada a uma sociedade global em que a intolerância e as desigualdades vêm aumentando, a partir de defesas particularistas. Em suma, o autor indica que uma cultura de apreciação dos acordos individuais e de abertura para a negociação entre os indivíduos desde uma relação inter partes não-maleficente propicia o desenvolvimento de uma relação extra partes, beneficente.

Por óbvio, esse posicionamento envolve uma perspectiva democrática, que entre indivíduos corresponde ao livre consentimento mútuo e no âmbito da governabilidade representa a negociação em busca de um acordo político equilibrado. Em certa medida, equivale a negociar alternativas intimistas, ainda que 
controladas, visando sua substituição da hegemonia do consenso.

Ainda que com uma "boa intenção", a hegemonia consensual falha por não levar em conta o aspecto particular da moral, assim como da fé, dentre outros aspectos culturais, reforçando assim a ideia da intolerância. Globalmente, como o próprio autor igualmente menciona, padrões de consentimento são mais fáceis de serem alcançados, mas resta aqui demonstrar que essa é uma falsa ilusão.

No entanto, a acepção da bioética contemporânea envolve discursos e práticas multidisciplinares. Nesse sentido, essa ciência procura trabalhar com a provisoriedade dos entendimentos, tendendo a romper com as decisões monopolizadas. Mesmo assim, a questão do livre consentimento permanece problemática diante dos grupos e indivíduos em situação de vulnerabilidade.

Outro ponto igualmente importante na questão da autonomia é o caso da utilização de material, inclusive biológico, e dados obtidos com a pesquisa. A finalidade antes taxativa, a partir da nova resolução passa a ser relativa, a depender do consentimento do participante.

Outras modificações foram sutis na linguagem, mas significativas no reconhecimento de direitos protetivos e compensatórios aos participantes. Exemplo disso está contido na alínea $o$, do item $I I I .2$, onde o antigo "ou" foi substituído pelo "e": "assegurar aos participantes da pesquisa as condições de acompanhamento, tratamento, assistência integral $\boldsymbol{e}$ orientação, conforme o caso, enquanto necessário, inclusive nas pesquisas de rastreamento". E em benefício econômico e intelectual da pesquisa no Estado, de forma ampla, a inclusão inédita de requisitos para as pesquisas em âmbito internacional ou envolvendo colaboração estrangeira exige, por exemplo, o registro obrigatório no Brasil de eventuais patentes de drogas desenvolvidas no território.

$\mathrm{Na}$ linha de revalorização da autonomia, as pesquisas envolvendo mulheres não grávidas, não as obrigam, após consentirem em participar da pesquisa, a fazer uso de contraceptivos. Já com relação à proteção em atenção ao princípio da nãomaleficência, para além da autonomia, os participantes possuem o direito de serem advertidos do que poderiam ser considerados meros desconfortos, para além dos eventuais riscos.

As principais diretrizes garantidoras da beneficência estão 
relacionadas às pesquisas médicas ou clínicas aplicadas a partir de pesquisas de medicamentos. Dentre as novidades trazidas pela resolução 466/12 estão: a garantia que o placebo, quando necessário, será comparado com os efeitos dos melhores métodos equivalentes existentes; o acesso gratuito e por tempo indeterminado ao melhor tratamento existente, independente dos resultados da pesquisa, isto é, seja ele oriundo dos resultados da própria pesquisa ou não. $\mathrm{O}$ tratamento deve ser iniciado mesmo no intervalo da pesquisa, devendo ser considerado um estudo de extensão e justificado pelo médico que acompanha o participante, sendo determinante a ética médica.

Em termos jurídicos, o processo de consentimento livre e esclarecido sofreu algumas alterações. Dentre delas, a inclusão do assentimento dentre as opções. $\mathrm{O}$ assentimento se consubstancia em um documento elaborado em linguagem acessível para os menores ou para os legalmente incapazes, por meio do qual, após os participantes da pesquisa ser devidamente esclarecidos, explicitam sua anuência em participar da pesquisa, sem prejuízo do consentimento de seus responsáveis legais. Esse documento se diferencia do TCLE por ser direcionado especificamente aos menores ou incapazes. Já o TCLE é o documento no qual é explicitado o consentimento livre e esclarecido do participante e/ou de seu responsável legal, de forma escrita, devendo conter todas as informações necessárias, em linguagem clara e objetiva, de fácil entendimento, para o mais completo esclarecimento sobre a pesquisa a qual se propõe participar, o adulto cidadão capaz.

\subsection{A importância dos comitês de ética em pesquisa}

Na perspectiva política, merece especial enfoque o papel dos CEPs aliados aos demais órgãos que com eles configuram o exercício de administrar o controle da ética da pesquisa científica no Brasil. Outro ponto fundamental dos CEPs para além das práticas políticas que se configuram, é o papel pedagógico que exercem sobre os pesquisadores, que muitas vezes impulsionados pela pesquisa e pelos investimentos não percebem e não conhecem a importância dos limites éticos e das implicações humanas, jurídicas e políticas que condicionam. O resultado da pesquisa realizada por Peres e Job (2010) entre alunos de medicina, aponta vulnerabilidade dos 
estudantes às propagandas das indústrias farmacêuticas, porém estas não chegam a alterar as percepções éticas atribuídas pelo Código de Ética médica, embora reconheça a necessidade do constante debate sobre o tema. Nesse ponto, os CEPs funcionam como importante arena de discussão, além de tudo, interdisciplinar.

Outro ponto que o estudo de Barbosa, Corrales e Silbermann (2014) destaca é a previsão na resolução 466/12, da elaboração de um complemento específico em termos resolutivos, próprios para as ciências sociais. Essa proposta resultaria na criação de uma resolução que daria às ciências sociais um sistema próprio, ligado ao Ministério da Ciência, Tecnologia e Inovação, afastada do biocentrismo, pois de matriz mais antropocêntrica. Na visão dos autores essa separação poderia comprometer a interdisciplinaridade e tornar praticamente inalcançável a transdiciplinaridade proporcionada pela união dos saberes nos comitês de ética regionais. De fato, uma nova resolução foi aprovada, dedicada às ciências sociais, a resolução 510/16, porém não trouxe mudanças significativas, gerando mais dúvidas do que soluções na comunidade científica.

Apesar das qualificações do sistema CEP/CONEP, essa problemática acerca da possível separação dos comitês em áreas, o que poderia comprometer a comunicação entre os saberes, ocorre politicamente também fora do âmbito acadêmico. Tensões que poderiam resultar em modificações cruciais no sentido de relativizar o controle realizado pelos órgãos, bem como dos princípios bioéticos, fazem parte de um novo movimento dentre as tentativas cíclicas de flexibilização da ética em pesquisa.

Há uma interessante discussão sobre a ameaça que a pesquisa voltada à justiça social, vem sofrendo por parte de grupos de interesses atuando através de propostas normativas que visam desequilibrar a relação entre pesquisadores e participantes das pesquisas, além de tentar comprometer seu viés democrático. Essa problemática merece ser aprofundada tendo em vista os avanços conquistados pelos estudos e comitês envolvidos na proteção da bioética em pesquisa.

Existe uma forte tendência à diminuição do atendimento ao requisito justiça e equidade. Essa afirmação não é precipitada, pois parte da análise de trabalhos publicados na área da saúde, como o estudo comparativo de Germano e França (2014), apontam a gradual 
diminuição das exigências éticas nas versões revisadas da Declaração de Helsinki, principalmente no que se refere ao equilíbrio entre riscos e benefícios que recaem sobre os participantes. A representação do Estado brasileiro nessas oportunidades sempre foi firme em defender a bioética, posicionando-se claramente contra as modificações que visam acatar interesses alheios à ética, seja ela baseada em princípios próprios do ser humano ou culturais.

No entanto, conforme Garrafa e Prado (2001), o fundamentalismo que se apresenta tem por base o quesito econômico e vem ignorando a vulnerabilidade social, enfatizada por Diniz e Corrêa (2011), aquela diferença provocada pela desigualdade econômica. Além disso, esse imperialismo ético ameaça a essência democrática e equânime com que as conferências e grupos como os comitês vêm desenvolvendo seus pareceres, desde um exercício democrático.

Os argumentos geralmente giram em torno da urgência e da diminuição de custos em detrimento da cautela promovida pela pluriparticipação na construção das diretrizes bases. Por essa ótica injusta, Hossne (2003), fundador da Sociedade Brasileira de Bioética, chama atenção para a possibilidade de exploração da vulnerabilidade do próprio pesquisador e que a diminuição de custos envolve a expectativa de ínfima busca por reparações indenizatórias, especialmente em países subdesenvolvidos. No mesmo estudo, o autor destaca minuciosamente o rigoroso processo de criação da resolução 196/96, e o esforço para legitimá-la através de extenso estudo a partir de consultas transdisciplinares e revisão de literatura bioética, para então após debates, ser apresentada e aprovada pelo Conselho Nacional de Saúde.

No cenário que se apresenta, o imperativo ético normativo deve proteger a autonomia do indivíduo. Mas, para isso, interessante abordagem parte do trabalho de Tomanik (2008) onde propõe que a norma, as resoluções, bem como as organizações sociais e os comitês devem promover a abertura da ética para a liberdade de reflexão, visando à autonomia do indivíduo, fugindo de fundamentalismos em todas suas formas e da justiça baseada tão somente no controle social. A partir desse encaminhamento é possível problematizar a questão de que a ética quando aproximada do sentido de autonomia, é dinâmica, e não rígida.

Desse modo, procura-se esclarecer que embora as recentes 
tendências em flexibilizar a ética em pesquisa com seres humanos pareçam focar na autonomia do participante, esta ocorre mais na tentativa de eximir os responsáveis por eventuais consequências negativas do que promover de fato a autonomia no seu sentido mais benéfico. A autonomia proposta explora a vulnerabilidade, desequilibra de forma injusta a relação posta, desloca o sentido de equidade para o sentido de igualdade que ignora a diferença, mantendo apenas a meia integridade dos princípios da não maleficência, da beneficência, ambos conectados à assistência, e ares de liberdade sustentados por uma falsa noção de autonomia.

Justifica-se esse argumento conclusivo no seguinte sentido: afastar a rigidez e manter a dinâmica da ética implica a participação democrática. Somente através de núcleos de discussão que construam entendimentos provisórios, porém constantes, é que se pode manter a revisão necessária para uma ética efetivamente compromissada com a justiça e a equidade. Nesse ponto, a atuação dos comitês continua sendo crucial para manter o fluxo contínuo de discussão sobre a ética. A lei é o instituto que por mais tempo fixa essa provisoriedade.

\section{APONTAMENTOS CRÍTICOS A PARTIR DA ANÁLISE DO TEXTO LEGAL DA PROPOSTA LEGISLATIVA 200/2015 E AS TENDÊNCIAS NA PESQUISA CLÍNICA}

O projeto de lei (PLS) n ${ }^{\circ} 200$ de 2015, proposto pelo Senado Federal será objeto de avaliação nesta etapa do presente estudo, no sentido de identificar nas suas diretrizes, tipologias modificativas e a quais princípios éticos estão relacionados. Desse modo, através da observação do texto legal foi possível identificar os conteúdos aparentemente mais polêmicos em duas vertentes: a que reúne as condições atreladas ao princípio da justiça e outra ligada a questão estrutural, no que parece ser a flexibilização do controle sobre a pesquisa. Há ainda um terceiro item que se aproxima do princípio da autonomia e em certa medida, relativiza a não maleficência: a possibilidade de remuneração dos participantes.

A proposta em tramitação encontra espaços de avanço na sua aprovação e gera interessantes discussões para se pensar o caminho percorrido pela bioética e o futuro da pesquisa envolvendo seres 
humanos no Brasil e no mundo. Os itens selecionados como de maior relevância para a discussão dessas novas tendências são, em um primeiro momento, aqueles que envolvem: o tratamento dos participantes e a utilização de placebo.

A diminuição dos custos da pesquisa, bem como a aceleração de prazos são dois critérios que justificam indiretamente os direcionamentos que serão daqui em diante abordados, exceto uma, que é a possibilidade de remuneração de participantes, mas que, no entanto, tendo em vista as demais alterações, parece que ainda assim, implicaria em um saldo positivo para o financeiro da pesquisa. A demora na avaliação e eventual aprovação dos projetos é um forte argumento da nova proposta.

Em atenção ao princípio da justiça ou, nesse caso, equidade dentre os atores da pesquisa, o tratamento gratuito aos participantes é administrado por tempo indeterminado e condicionado ao melhor tratamento disponível. Na proposta legislativa, a continuidade do tratamento pós-ensaio clínico pode ser interrompida, dentre outros casos, após dois anos em que o medicamento estiver sendo regularmente comercializado no país. Esse novo equilíbrio baseia-se na igualdade absoluta, e não na equidade com base na diferença. $\mathrm{O}$ tempo é limitado, mas suficiente para que o participante se submeta a pesquisa em função do tratamento.

O projeto prevê a não diferenciação do uso de placebo nos casos em que há método e também nos casos em que não há, ou seja, a partir do produto de comparação. Por ora, o uso de placebo é permitido de maneira limitada, autorizando-se seu uso em comparação com os estudos em que já existe método comprovado. No geral, o uso irrestrito de placebo, restou consolidado como opção excepcional diante de doença ou outra enfermidade para qual não haja nenhum tratamento disponível. O custo da aprovação de medicamentos em função da lógica mercadológica oferece um grande risco à saúde pública.

No que tange a sistemática estrutural e rigorosa de controle das pesquisas no Brasil, efetuada pelos comitês de éticas em pesquisa vinculados ao Ministério da Saúde, e seus critérios, tendem a ser flexibilizados. Em nome da não utilização de critérios diferenciados direcionado as pesquisas, a estrangeira não seria mais diferenciada da pesquisa nacional, autorizando-se seu registro em outro país. No 
entanto, exceção a essa isonomia ocorreria para estudos considerados de maior relevância. Estes passariam a ter prioridade de análise pelo sistema.

O Sistema Nacional de Ética em Pesquisa Clínica ficaria vinculado à Agência Nacional de Vigilância Sanitária (ANVISA), registrando os comitês em ética na pesquisa que poderiam ser institucionais ou independentes. Por fim, abre exceções a proibição de remuneração, quando não houver benefícios terapêuticos diretos aos participantes de pesquisa, mediante inclusão do indivíduo em cadastro nacional de participantes. Nesse caso, não pode participar em mais de uma pesquisa, e respeitado um intervalo de no mínimo seis meses entre uma e outra no caso de pesquisas clínicas com dose elevada de tratamento. Porém, esse tipo de pesquisa remunerada só possa ser feita, conforme texto legal, nos chamados ensaios clínicos de fase $I$ ou fase inicial.

Se aprovada, a proposta trará modificações significativas ao contexto brasileiro de pesquisa envolvendo seres humanos, demonstrando uma tendência a relativizar os princípios bioéticos, afastadas da atual realidade em pesquisa clínica no país. Os principais pontos conflituosos atingem justamente as questões jurídicas e surpreendentemente tendem a flexibilizar a pesquisa clínica, representando para alguns estudiosos um recuo, por ignorar os históricos conhecidos de experimentações em humanos e a experiência política e pedagógica das sugestões do sistema CEP/CONEP. A aceitação do projeto de lei implica na rediscussão sobre os princípios éticos.

\section{CONCLUSÃO}

A problemática da proteção da pesquisa envolvendo seres humanos nos requisitos éticos e bioéticos é motivo de constante preocupação da comunidade científica. O conflito de interesses é constante.

A presente abordagem procurou demonstrar brevemente recortes históricos, especialmente através da construção regulamentadora da disciplina ética, retratando partes do contexto maior que envolve a bioética no cenário nacional. Com isso buscou ao mesmo tempo vislumbrar o pano de fundo dessas questões, no 
plano teórico e legislativo.

No plano resolutivo, constatou avanços em medidas protetivas de caráter ético-científico nas pesquisas, em especial envolvendo seres humanos clinicamente. Igual avanço pode ser constatado na segurança jurídica exigida através de documentos, como o Termo de Consentimento Livre e Esclarecido (TCLE).

Em contrapartida, foi possível adentrar ainda que brevemente nas propostas não consolidadas, mas tendentes a flexibilizar as estruturas e relativizar os direitos conquistados, buscando a eficiência das demandas em pesquisa. Para alguns pesquisadores, isso pode significar uma ameaça à proteção dos participantes em pesquisa e aos limites éticos impostos desde o pós-segunda guerra, construído de forma democrática e plural.

Possíveis alterações no sistema CEP/CONEP demonstram intenções que afetam a cultura promovida pelo papel dos CEPs institucionais e tornam incerta a perspectiva de não influência econômica direta no âmbito da pesquisa clínica. Longe de finalizar o debate, esse ensaio pesquisa procurou antes provocar mais perguntas do que oferecer respostas.

Corresponder aos critérios de pesquisa consolidados no Brasil nem sempre é tarefa fácil, porém deve buscar o comprometimento do pesquisador com a postura ética de respeito ao participante e a dignidade da pessoa humana, conforme prevê a Constituição brasileira. Nessa seara, a comunidade jurídica precisa estar atenda as possíveis mudanças especialmente no que tange a proteção dos envolvidos, inclusive os próprios pesquisadores.

A participação nos processos de aprovação dos projetos de pesquisa, em reuniões multidisciplinares e transdisciplinares democraticamente resultam em equilíbrios consensuais plurais, pois a partir de múltiplas visões. Embora alguns aspectos necessitem ser estudados caso a caso, as diretrizes gerais continuam sendo fundamentais ao respeito para com os direitos humanos, complementadas pela atuação dos comitês regionais de ética, atendendo aos contextos locais.

De qualquer modo, faz-se necessário convocar a manifestação da sociedade científica e dos cidadãos em geral sobre as possíveis consequências ético-jurídicas com relação à aprovação de legislação pertinente. A proteção dos participantes, nas previsões de utilização 
do polêmico placebo, a remuneração dos participantes, a continuidade do tratamento pós-ensaio clínico, dentre outras questões apontadas merecem cautela interventiva, tendo em vista o possível desmantelamento do sistema CEP/CONEP em virtude de uma legislação genérica e tendente a perspectivas éticas nebulosas.

\section{REFERÊNCIAS BIBLIOGRÁFICAS}

BARBOSA, Adriana Silva; CORRALES, Carlos Montero; SILBERMANN, Marcos. Controvérsias sobre a revisão ética de pesquisas em ciências humanas e sociais pelo Sistema CEP/Conep. Rev. Bioética, v. 3, n. 22, p. 482-92, 2014.

DINIZ, Debora; CORRÊA, Marilena. Declaração de Helsinki: relativismo e vulnerabilidade. Cad. Saúde Pública, Rio de Janeiro, v. 17, n. 3, p. 679-688, 2001.

ENGELHARDT Jr., H. Tristram. Fundamentos da Bioética. São Paulo: Loyola. 1998. Capítulo 3. "Os princípios da bioética", p. 131-168.

GARRAFA, Volnei; PRADO, Mauro Machado do. Mudanças na Declaração de Helsinki: fundamentalismo econômico, imperialismo ético e controle social. Cad. Saúde Pública, Rio de Janeiro, n. 17, v. 6, p. 1489-1496, 2001.

GOLDIM, José Roberto. O consentimento informado numa perspectiva além da autonomia. Revista AMRIGS, Porto Alegre, v. 3,4, n. 46, p. 109-116, 2002.

HOSSNE, William Saad. O poder e as injustiças nas pesquisas em seres humanos. Interface - Comunic., Saúde, Educ., v. 7, n. 12, p. 55-70, 2003.

MARTÍNEZ, Gabriela Rueda; MONSORES DE SÁ, Natan. Impacto de laausenciadelConsejo Nacional de Bioética Colombiano. Revista Latinoamericana de Bioética, v. 2, n. 29, p. 144-155, 2015.

PERES, Gabriel; JOB, José Roberto Pretel Pereira. Médicos e Indústria Farmacêutica: Percepções Éticas de Estudantes de Medicina. Revista Brasileira de Educação Médica, v. 34, n. 4, p. 515-524, 2010.

SANTANA, Clarissa Cerqueira de; FARIA, Juliano Almeida de. A pesquisa com seres humanos nos periódicos da área de contabilidade. In.: Anais - X Congresso Brasileiro de Bioética. Revista da Sociedade Brasileira de Bioética, Vol. 9, Suplemento, 2013.

TOMANIK, Eduardo Augusto. A ética e os comitês de ética em pesquisa 
com seres humanos. Psicologia em Estudo, Maringá, v. 13, n. 2, p. 395-404, 2008.

ZOBOLI, Elma Povone. "Contribuição da bioética para a evolução da investigação científica: ponte entre sabedoria e conhecimento". In: Bioética ou Bioéticas na evolução das sociedades. Coordenado por Maria do Céu Patrão Neves e Manuela Lima. Edição luso-brasileira. Coimbra: Gráfica de Coimbra, p. 43-55, 2005. 
